\title{
Experimental verification of a high performed multiple-band metamaterial absorber
}

\author{
Nanjie Li, Dawei Liu \\ School of Electrical and Electronic Engineering, Huazhong University of Science and \\ Technology, Wuhan, China 430000
}

\begin{abstract}
In this paper, a thin-film metamaterial absorber with multiple-band is experimental verification and simulated analysis. Two high absorption peaks higher than $99 \%$ and $98 \%$ are obtained at normal incidence. Resonance of the local surface plasma mode and the internal surface plasmon mode lead to the two high absorption peaks. The impedance matched condition is obtained behind two high absorption peaks. Measured results indicate that high absorption performed can be observed different dielectric layer combinations $\left(\mathrm{Al}_{2} \mathrm{O}_{3}-\mathrm{ZnSe}, \mathrm{Al}_{2} \mathrm{O}_{3}-\mathrm{Al}_{2} \mathrm{O}_{3}\right.$, and $\left.\mathrm{ZnSe}-\mathrm{ZnSe}\right)$. The measured metamaterial absorber also shows high absorption under both TE and TM configurations at various incident angles.
\end{abstract}

Keywords: Photo-electrochemical, metamaterial, absorber

\section{Introduction}

Metamaterials are artificial engineered and manufactured materials which can exhibit unique electromagnetic properties that can't be obtained in nature[1-2]. Metamaterial shows a wide range of potential applications, such as ultrafast modulators, highly sensitive sensors, and antenna systems [3-5]. In these potential applications, the absorption performance of metamaterial arouses the interest of researchers. Since 
Landy et al [6] designed and verified a single-band perfect absorber which consists of cutting wires and metallic split ring resonators, metamaterial absorber becomes a hot research area [7-10]. Subsequently, many novel structures are proposed and preparation to achieve metamaterial absorbers, which are insensitive to polarization and incident angle [11]. Metamaterial absorbers can be applied in a wide range due to their unique properties, including thermal emitters, solar cells , and imaging devices [12-17]. In these proposed metamaterial absorbers, near-unity absorbance is characterization through modulation the magnetic permeability $\mu$ and electric permittivity $\varepsilon$. However, many proposed designs are single-band metamaterial absorbers [18-21]. Though multi-dielectric layers (using the same of dielectric layers) metamaterial absorber is fabricated with a absorption band [22]. Recent years, considerable effort is performed to achieve multiple-band metamaterial absorber [23-24]. These proposed absorber are designed through optimizing structure design to obtain multiple-band [25-28]. However, the dielectric layer, and especially its dielectric layer combination, are two important but often not being taken seriously factors in designing multiple-band metamaterial absorber.

In this paper, a dual-band metamaterial absorber is proposed and manufactured through optimal arrangement dielectric layer combinations. Two resonance modes are found through calculating electric field intensity distributions: the local surface plasma (LSP) mode and the internal surface 
plasmon (ISP) mode. In previous work [29], it is found that the resonance of LSP modes results in the transmission reducing and maybe leads to the absorption peak. In this paper, the feasibility is confirmed. Moreover, simulated results also indicate that the ISP mode provides important contribution in exciting the short- and long-wavelength absorption peaks.

\section{Materials and methods}

2.1 Structural design and methods

In detail, the proposed dual-peak metamaterial absorber consists of alternating of flat metal layers and dielectric layers, as shown in Fig 1(a-b). In the proposed structure, the metal layer is made of gold, and the dielectric layer is made of different materials $\left(\mathrm{Al}_{2} \mathrm{O}_{3}\right.$, or $\left.\mathrm{ZnSe}\right)$. All of structural parameters are: $\mathrm{P}=20 \mu \mathrm{m}, \mathrm{R}=3 \mu \mathrm{m}, \mathrm{w}=2 \mu \mathrm{m}, \mathrm{H}=\mathrm{h}=0.35 \mu \mathrm{m}$. The measured absorption spectra and optical photo of samples are shown in Fig 1(c-d). Simulations are performed by the Ansofts HFSS 13.0. Ideal electromagnetic conductor planes are adopted on the designed unit cell normal to the $\mathrm{x}$ or $\mathrm{y}$ axises. The designed unit cell is tested in air under the electromagnetic waves incident along the $\mathrm{z}$ axis. Due to the thickness of the bottom gold layer is larger than the skin depth of electromagnetic waves, the transmitted of samples closes to zero. Thence, the absorption of the proposed metamaterial absorber can be obtained as:

$$
\mathrm{A}(f)=1-\mathrm{R}(f)
$$

In simulations, the gold layer is given by the Drude model, the damping 
constant is set to $\omega=4.08 \times 10^{13} s^{-1}$, and the plasma frequency is set to $\omega_{p l}=1.37 \times 10^{16} s^{-1}$ [30]. The permittivity of dielectric layer $\mathrm{ZnSe}$ is given 5.74 and $\mathrm{Al}_{2} \mathrm{O}_{3}$ is given $2.28[31,32]$. The ground dielectric layer is given as SU-8 [33]. Four ideal electric-magnetic conductor planes are used on boundaries of the unit cell [34].

\subsection{Experimental methods and simulation methods}

Samples of the proposed metamaterial absorber are fabricated as following: A silicon wafer is selected firstly to be a substrate. A gold layer is deposited on the selected silicon wafer by using low pressure chemical vapor deposition. A SU8 layer is spin on the first silver layer as a basic dielectric layer by using MSC-400Bz-6N spinner. Then, the rest of three gold layers ( the preparation method is the same as above) and two dielectric layers (a $\mathrm{ZnSe}$ layer and an $\mathrm{Al}_{2} \mathrm{O}_{3}$ layer) are deposited alternately. The $\mathrm{ZnSe}$ layer and the $\mathrm{Al}_{2} \mathrm{O}_{3}$ layer are deposited on gold layer by plasma-enhanced chemical vapor deposition. The samples of the proposed metamaterial absorber are defined by using the regular electron-beam lithography. The area of samples is $2 m m \times 2 m m$. The measured absorption spectra is obtained by using the Bruker Optics Equinox 55 Fourier spectrometer. The optical images are obtained by using optical microscope. These samples in Fig 1(d) are named as " $\mathrm{ZnSe}-\mathrm{Al}_{2} \mathrm{O}_{3}$ " dielectric layer combinations. 

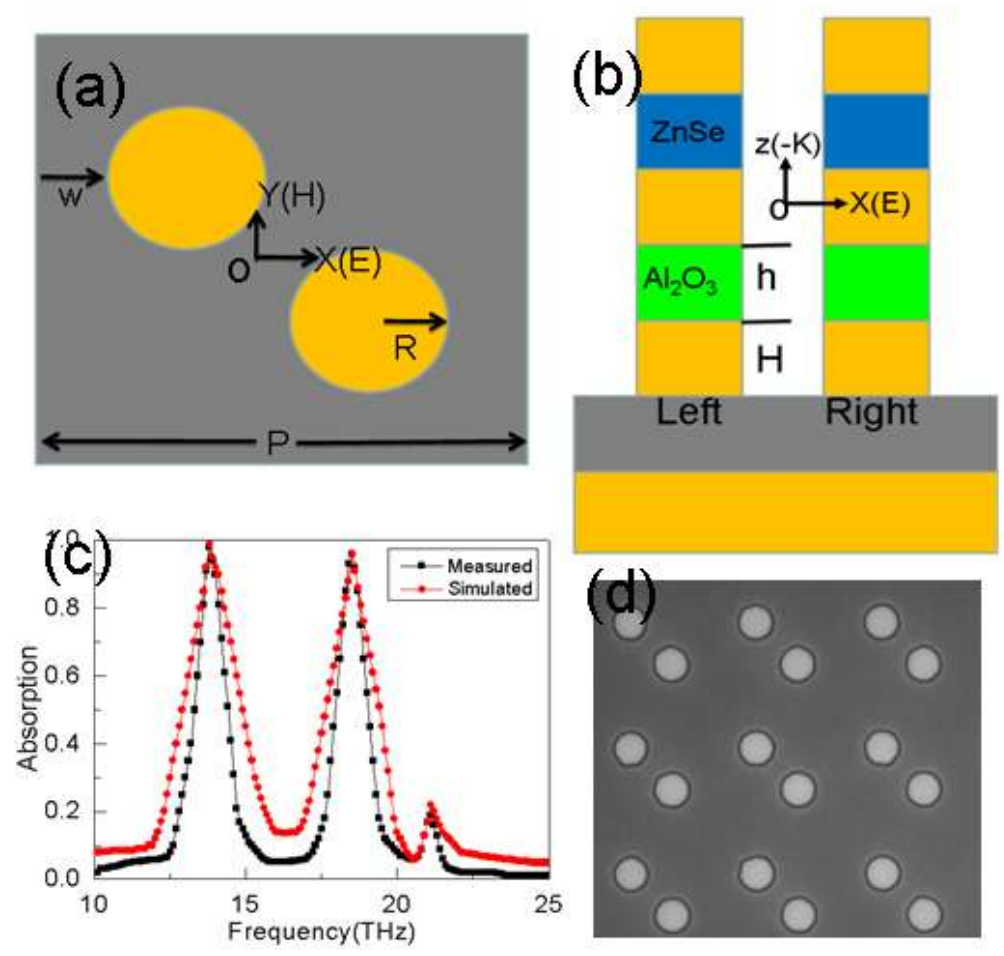

Fig.1. (a) Top view of a unit cell. (b) Side view of a unit cell on the xoz plane.

The orange part is gold layer, the gray part is SU-8 layer, the blue part is $\mathrm{ZnSe}$ layer, the green part is $\mathrm{Al}_{2} \mathrm{O}_{3}$ layer. (c) Measured and simulated absorption spectra. (d) optical images of samples.

\section{Results and discussions}

For a deeper understanding of the physical mechanisms of dual absorption peaks, electric field intensity distributions are calculated at these resonance frequencies, as shown in Fig 2. For the low-frequency absorption peak at $13.8 \mathrm{THz}$, two kinds of resonance modes can be observed. One kind of resonance mode is local surface plasma (LSP), which is excited on edges of circular gold layers. Moreover, the coupled and interacted of LSP modes in the middle circular dielectric layer is found, as shown in Fig 2(a). Another kind of resonance mode is internal surface plasmon (ISP) in the top circular dielectric 
layer, as shown in Fig 2(a). These resonance modes result in the low-frequency absorption peak. For the high-frequency absorption peak at $18.5 \mathrm{THz}$, there are also two kinds of resonance modes can be observed. One kind of resonance mode is also the LSP modes, which are excited on edges of circular gold layers. Moreover, the coupled and interacted of LSP modes is found in the top circular dielectric layer, as shown in Fig 2(b). Another kind of resonance mode is the ISP mode in the middle circular dielectric layer, as shown in Fig 2(b). These resonance modes excite the long-wavelength absorption peak. It is interesting that the different of resonant positions of LSP and ISP modes leads to different absorption peaks (low- or high-frequency absorption peak). For the small absorption peak at $21.1 \mathrm{THz}$, only LSP modes can be found. Moreover, the intensity of of the coupled and interacted of LSP modes in the top circular dielectric layer is weaker than that in Fig 2(b), as shown in Fig 2(c). These LSP modes result in the small absorption peak at 21.1THz.
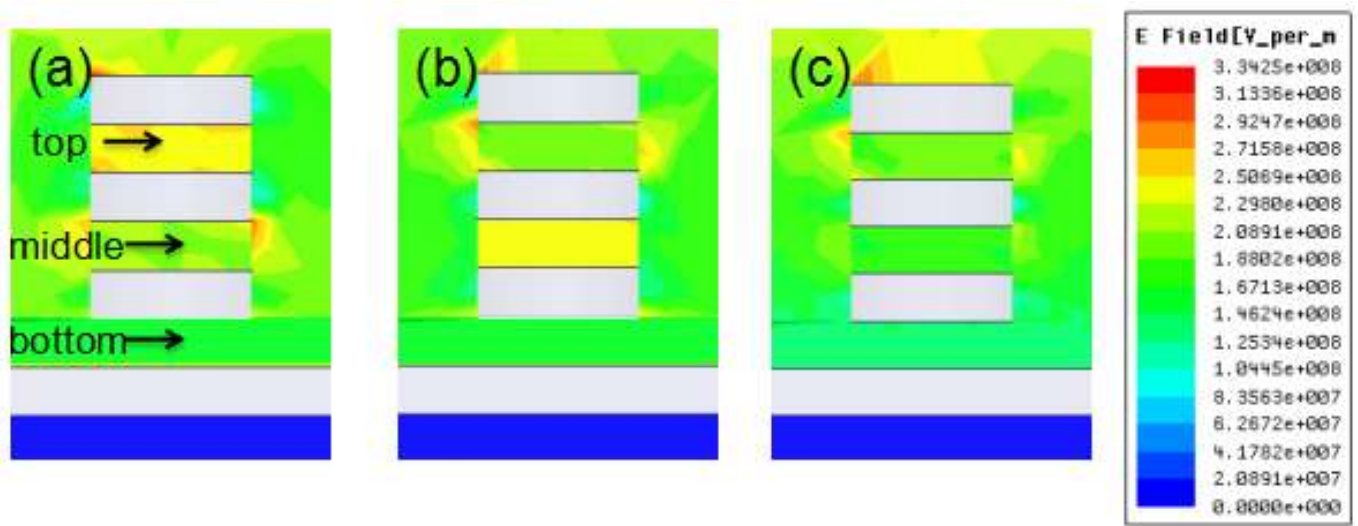
Fig. 2. Electric field distributions (in the case of the left circular stack, the right one is similar and not shown): (a) at $13.8 \mathrm{THz}$, (b) at $18.5 \mathrm{THz}$, (c) at 21.1THz.

The reflection of the proposed absorber is possible to be minimized and then the absorption is also to be maximized by adjusting the electric-magnetic resonances of the proposed structure [35]. To reveal the electromagnetic resonance properties behind dual absorption peaks, the $\mathrm{S}$ parameters retrieval method is adopted to extract the effective permittivity and permeability [36]. As shown in Fig 3(a), the real part of $\mu$ crosses zero at $f=13.8 \mathrm{THz}$ and at $f=18.5 \mathrm{THz}$, respectively. At the same time, the real part of permittivity also crosses zero at $f=13.8 \mathrm{THz}$ and at $f=18.5 \mathrm{THz}$, respectively, as shown in Fig 3(b). Both the the real parts of $\mu$ and permittivity cross zero at these same resonance frequencies, which leads to impedance matched conditions between the proposed absorber and the air interface achieve [37]. These electromagnetic resonance behaviors are very similarly to the previous literature [37]. 


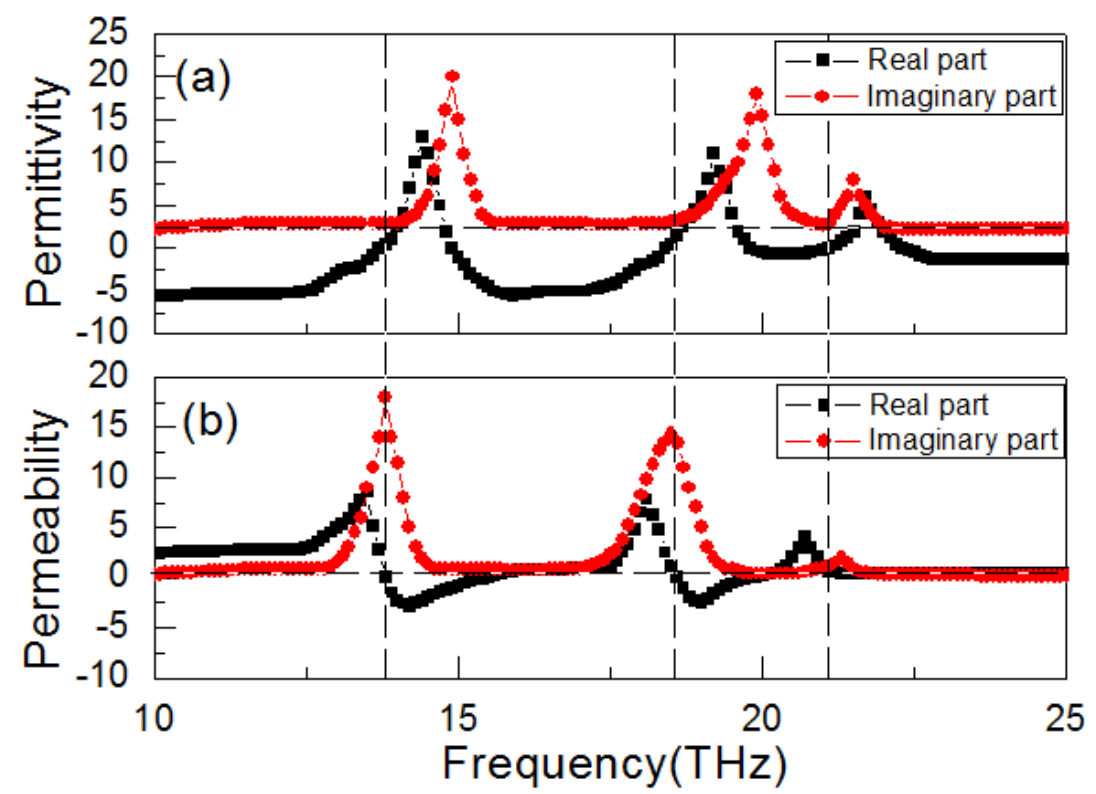

Fig 3. (a) The effective permittivity of the designed metamaterial absorber. (b) The effective permeability of the designed metamaterial absorber.

In order to reveals the relationship between the absorption performance of the designed metamaterial absorber and the dielectric layer combination, the dielectric layer combination in Fig 1(b) will be replaced by different dielectric layer combinations $\left(\mathrm{Al}_{2} \mathrm{O}_{3}-\mathrm{ZnSe}, \mathrm{Al}_{2} \mathrm{O}_{3}-\mathrm{Al}_{2} \mathrm{O}_{3}\right.$, and $\left.\mathrm{ZnSe}-\mathrm{ZnSe}\right)$. Fig 4 shows the absorption spectrum of the designed metamaterials absorber under the same structural parameters. For the $\mathrm{Al}_{2} \mathrm{O}_{3}-\mathrm{ZnSe}$ combination, the low-frequency peak is reduced to $91 \%$, and the high-frequency peak is reduced to $88 \%$. When the $\mathrm{Al}_{2} \mathrm{O}_{3}-\mathrm{ZnSe}$ combination is replaced by the $\mathrm{Al}_{2} \mathrm{O}_{3}-\mathrm{Al}_{2} \mathrm{O}_{3}$ combination, maximum absorption rates are reduced to $80 \%$ and $78 \%$, respectively. Finally, for the case of the $\mathrm{ZnSe}-\mathrm{ZnSe}$ combination, maximum absorption rates are reduced to $77 \%$ and $75 \%$, respectively. For these four dielectric layer combinations, resonance 
frequencies of dual absorption peaks are almost unchanged, which implies that the energy loss around resonance wavelengths are not related to the dielectric layer combinations.

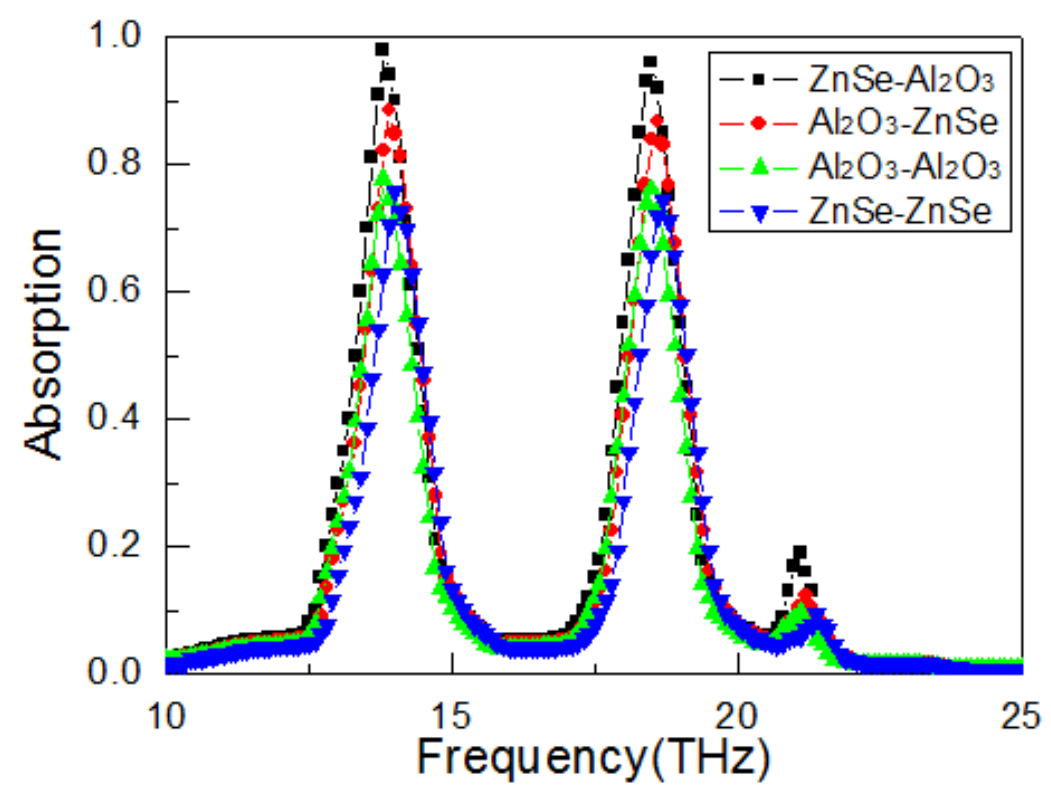

Fig 4. Measured absorption spectrum of different dielectric layer combinations

To understand relationship between the energy loss around resonance wavelengths and dielectric layer combinations $\left(\mathrm{ZnSe}-\mathrm{Al}_{2} \mathrm{O}_{3}, \mathrm{Al}_{2} \mathrm{O}_{3}-\mathrm{ZnSe}\right.$, $\mathrm{Al}_{2} \mathrm{O}_{3}-\mathrm{Al}_{2} \mathrm{O}_{3}$, and $\mathrm{ZnSe}-\mathrm{ZnSe}$ ), electric field distributions are calculated at 13.8THz and $18.5 \mathrm{THz}$, as shown in Fig 5 and Fig 6. For the low-frequency absorption peak with different circular dielectric layer combinations, two kinds of resonance modes can be observed (the LSP mode and the ISP mode). The resonant positions of LSP modes and ISP modes are the same with different circular dielectric layer combinations. It should be indicated that the variations of the intensity of LSP and ISP modes are similar to the maximum values of the low-frequency peak in Fig 4. For the high-frequency absorption peak, the LSP and ISP modes are also can be observed with different circular dielectric 
layer combinations, as shown in Fig 6. The intensity of LSP and ISP modes are reduced with different circular dielectric layer combinations, which is corresponding to the maximum values of the high-frequency peak in Fig 4. However, it should be indicated that the resonance positions of these resonance modes are different. For the low-frequency absorption peak, the coupled and interacted of LSP modes only occur between the middle and bottom circular gold layers, and ISP modes are observed in the top circular dielectric layer, as shown in Fig 5. For the high-frequency absorption peak, the coupled and interacted of LSP modes only occur between the top and middle circular gold layers, and ISP modes are observed in the middle circular dielectric layer, as shown in Fig 6.

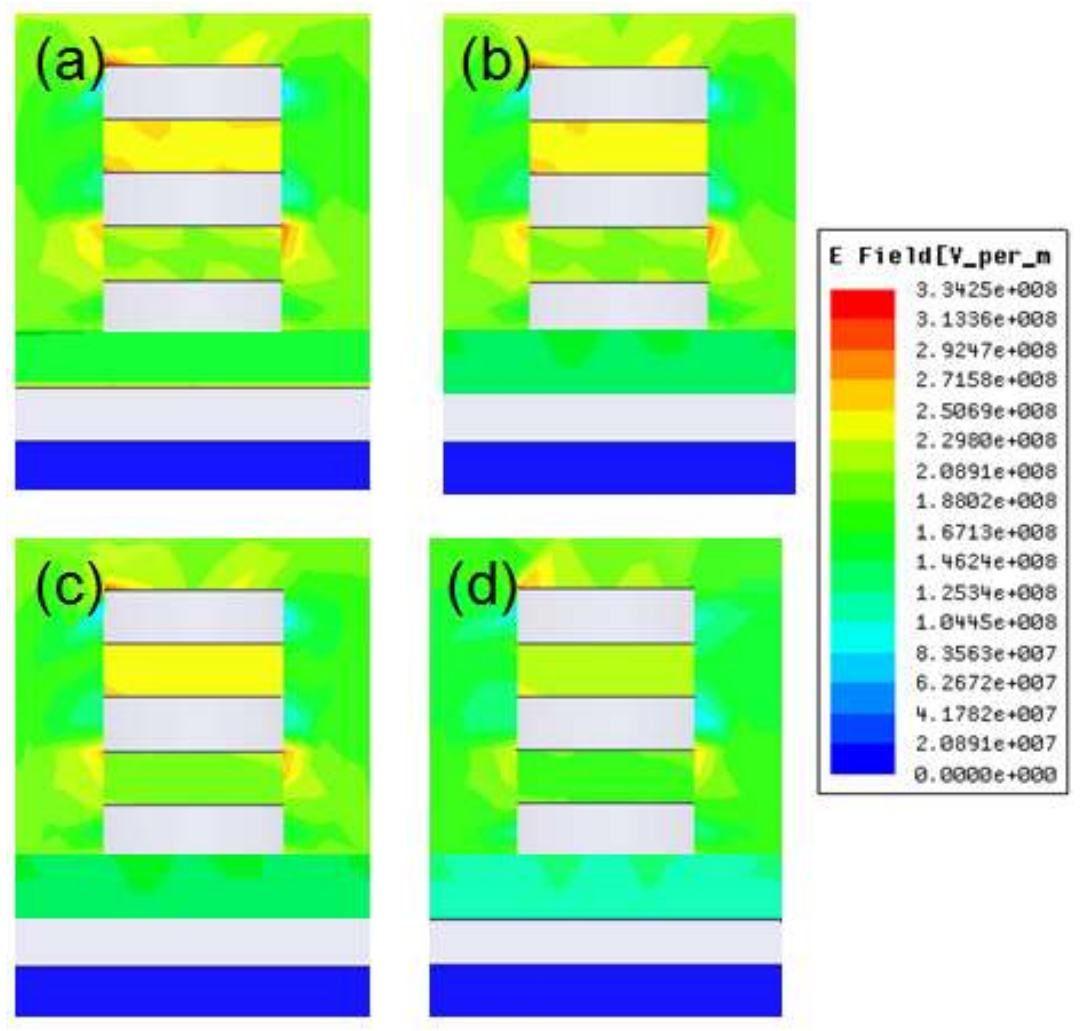

Fig 5. Electric field distributions of the short-wavelength absorption peak with different circular dielectric layer combinations (in the case of the left circular 
stack, the right one is similar and not shown).
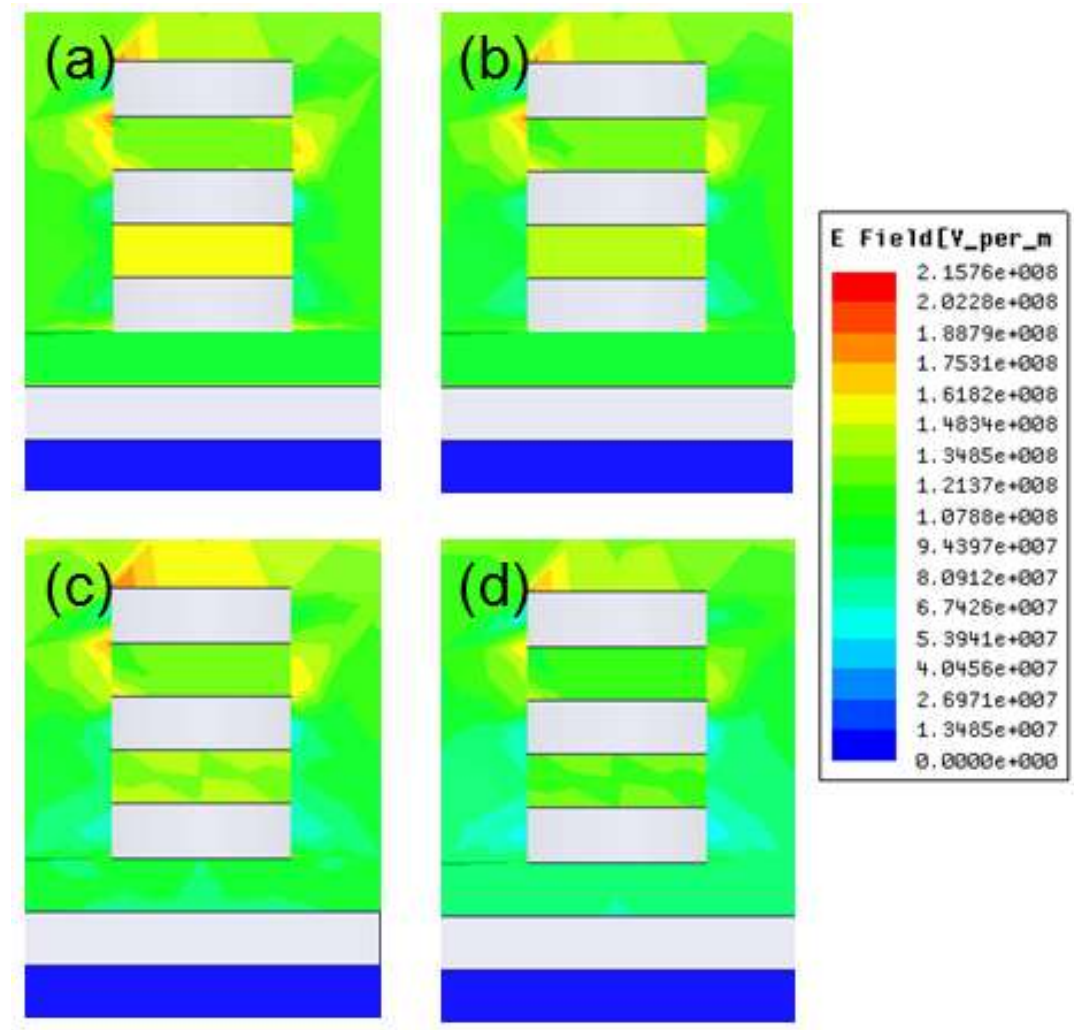

Fig 6. Electric field distributions of the long-wavelength absorption peak with different circular dielectric layer combinations (in the case of the left circular stack, the right one is similar and not shown).

To reveal the electromagnetic resonance behaviors with different circular dielectric layer combinations, effective permittivity and permeability are extracted by $\mathrm{S}$ parameters retrieval method. It is obviously that electromagnetic resonance behaviors with different circular dielectric layer combinations are similarly. However, the intensity of the electromagnetic resonance behaviors are reduced with different circular dielectric layer combinations. Moreover, positions of the crossing zero of the effective permittivity and permeability are not the same. For the 
$\mathrm{Al}_{2} \mathrm{O}_{3}-\mathrm{ZnSe}$ dielectric layer combination, the position of crossing zero of the effective permittivity shows a shifted to lower frequency, which is not consists with that of the effective permeability. Thence, the perfect impedance matching is reduced, which leads to the short- and high-frequency peaks reduce, as shown in Fig 4. Similarly, for the $\mathrm{Al}_{2} \mathrm{O}_{3}-\mathrm{Al}_{2} \mathrm{O}_{3}$, and $\mathrm{ZnSe}-\mathrm{ZnSe}$ dielectric layer combinations, the position of crossing zero of the effective permittivity shows a shifted to higher frequency, the perfect impedance matching is also reduced, and the maximum values of the low- and high-frequency peaks are lost.

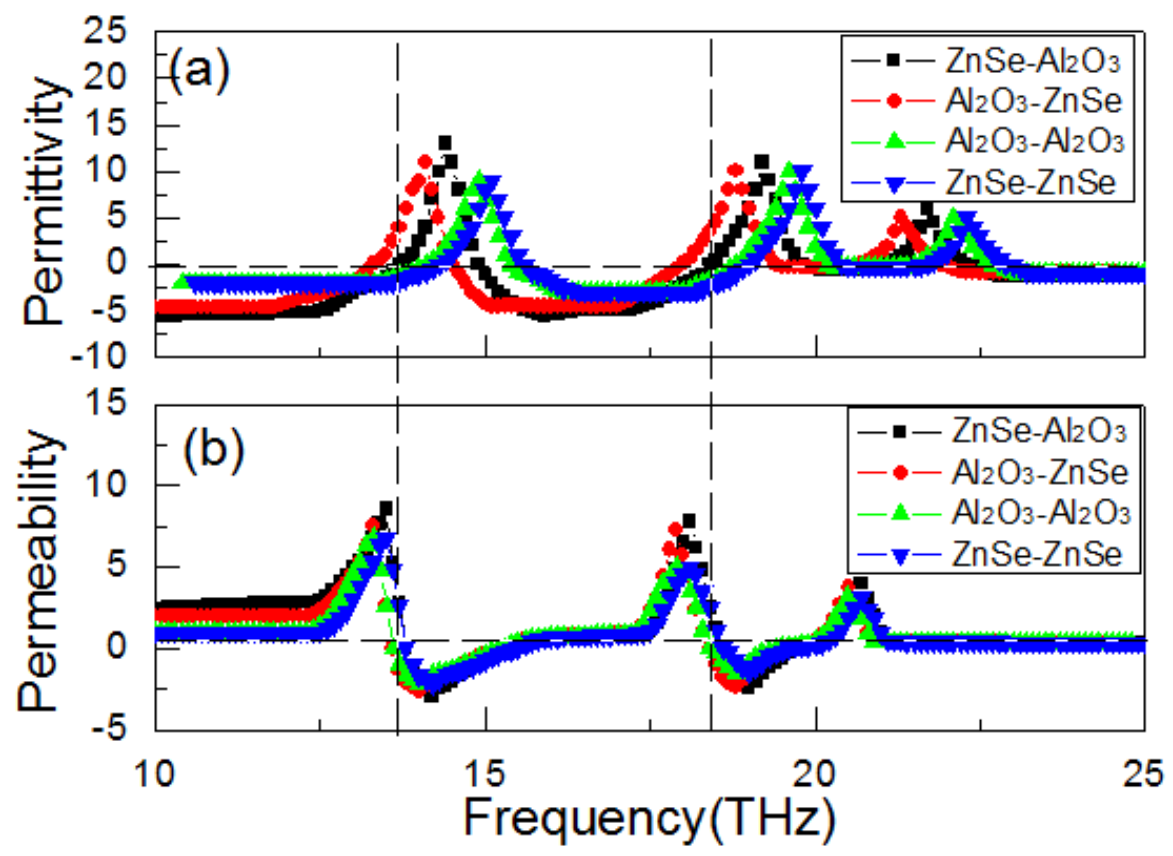

Fig 7. The effective permittivity and permeability with different circular dielectric layer combinations

To understand the mechanism the absorption peak at $21.1 \mathrm{THz}$, electric field distributions are also calculated, as shown in Fig 8. It is different that only one kind of resonance mode can be found: LSP modes, which are excited on 
edges of the top and middle circular gold layers. Moreover, the couple and interfered between LSP modes in the top circular dielectric layer is also observed, as shown in Fig 8. These results indicate that the small absorption peak at $21.1 \mathrm{THz}$ is excited by these LSP modes. It should be note that the resonance of ISP mode in the circular dielectric layer can't be found, which is an obviously difference with Fig 5 and 6. Moreover, the intensity of electric fields and coupling is weaker than that of the low- and high-frequency absorption peak, as shown in Fig 5 and 6. This is also can be found in Fig 7 that the electromagnetic resonance strength of the small absorption peak is weaker than the low- and high-frequency absorption peaks. Comparing the results of the Fig 5, Fig 6, and Fig 8, it can be found that the resonance of ISP mode in the top (Fig 5) or middle (Fig 6) circular dielectric layer provides the main contribution on the excited of the short- and long-wavelength absorption peaks. 


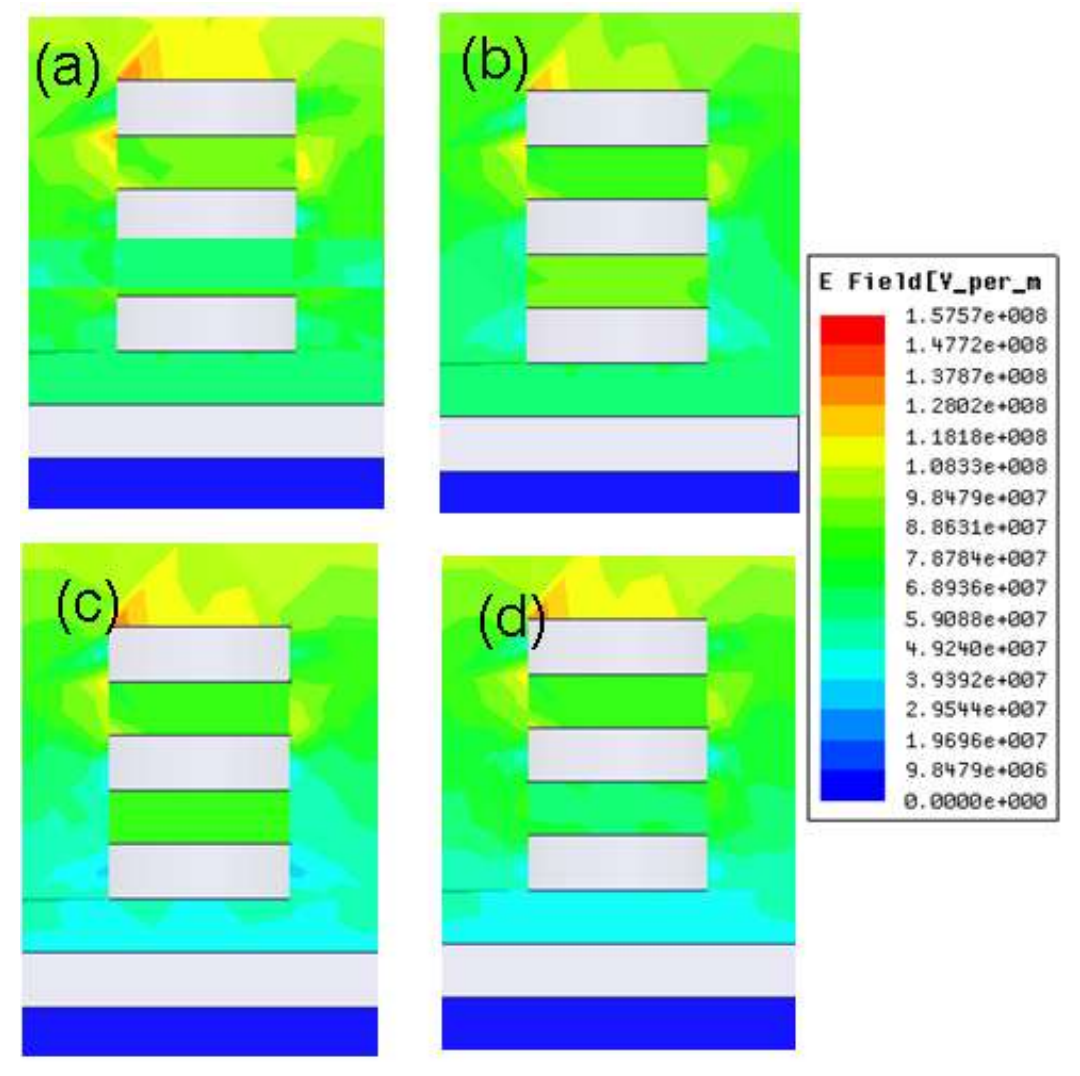

Fig 8. Electric field distributions of the small absorption peak with different circular dielectric layer combinations (in the case of the left circular stack, the right one is similar and not shown).

The designed metamaterial absorber can be applied in various optical devices, including thermal emitters and detectors, photovoltaic cells[38-39]. It is an important feature that absorbing as much of electromagnetic waves as possible for the designed metamaterial absorber. In this paper, absorption spectrum of the designed metamaterial absorber under both TE and TM configurations at various incident angles are measured, as shown in Fig 9. It is obviously that dual absorption peaks show a shifted to lower frequency when the incident angle is increased. However, differences still exists between Fig 9(a) and (b). For the TM 
configuration, a high absorption rate is also can be obtained when the incident angle reaches to $60^{\circ}$, as shown in Fig 9(a). While for the TE configuration, absorption rates are reduced obviously when the incident angle reaches to $60^{\circ}$, as shown in Fig 9(b). The abnormal decreased of dual absorption peaks is because that the magnetic field can't drive incident waves efficiently at a large incident angle [40].

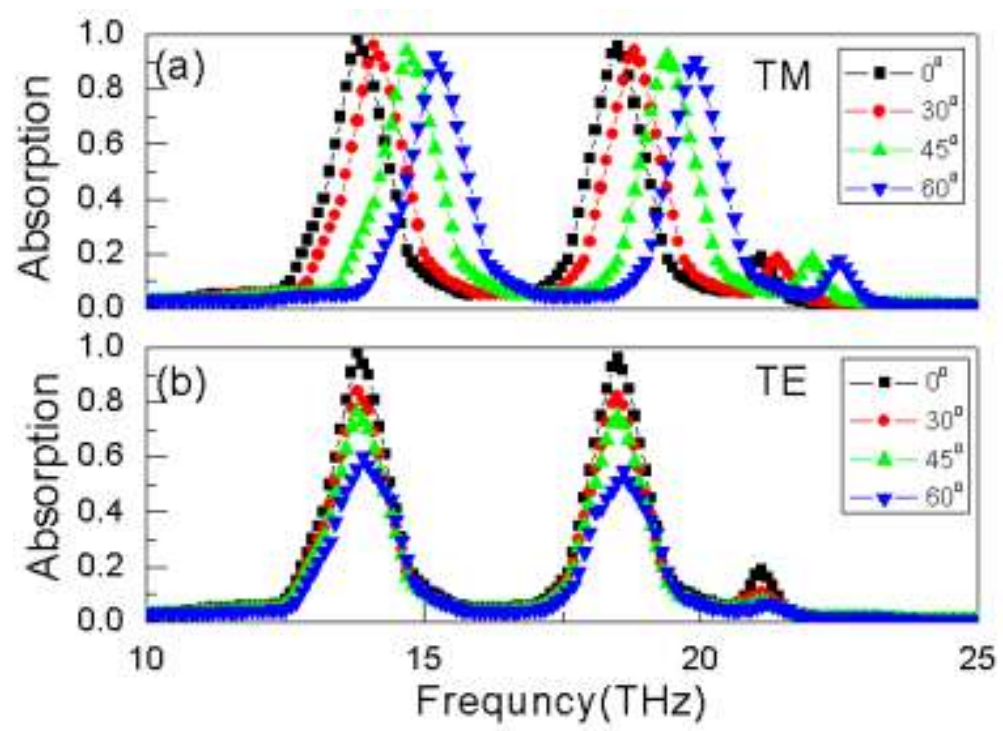

Fig 9. Measured spectrum of the designed metamaterial absorber under both TE and TM configurations.

\section{Conclusion}

In conclusion, a dual-band metamaterial absorber is experimental verification and simulated. Absorptivity of higher than $99 \%$ and $98 \%$ is obtained at normal incidence. Electric field distributions indicate that the low- and high-frequency are both excited by the resonance of the local surface plasma (LSP) mode and the internal surface plasmon (ISP) . However, for these peaks, the resonance positions of the ISP mode are different. 
For the low-frequency peak, resonance positions of the ISP mode are in the top circular dielectric layer. For the high-frequency peak, the resonance positions are in the middle circular dielectric layer. Moreover, the designed metamaterial absorber works well in the angular range from $0^{\circ}$ to $60^{\circ}$ under both TE and TM configurations.

Acknowledgements: This research is financially supported by National Natural Science Foundation of China(63455971)

\section{References}

[1] T. C. Choy, Effective Medium Theory, Principles and Applications; Oxford University Press: New York, 1999.

[2] W. Cai, V. Shalaev, Optical Metamaterials: Fundamentals and Applications; Springer: New York, 2009

[3] H. T. Chen, W. J. Padilla, M. J. Cich, A. K. Azad, R. D. Averitt, A. J. Taylor, "A metamaterial solid-state terahertz phase modulator", Nat. Photonics 2009, 3, 148-151.

[4] J. N. Munday, H. A. Atwater, "Large Integrated Absorption Enhancement in Plasmonic Solar Cells by Combining Metallic Gratings and Antireflection Coatings", Nano Lett. 11 2195-2200(2011)

[5] D. Dregely, R. Taubert, J. Dorfmuller, R. Vogelgesang, K. Kern, H. Giessen, “3D optical Yagi-Uda nanoantenna array”, Nat. Commun. 2011, $2,267$. 
[6] N. I. Landy, S.Sajuyigbe, J. J. Mock, D. R. Smith, W. J. Padilla, “Perfect metamaterial absorber "Phys. Rev. Lett. 2008, 100, 207402 [7] L. Huang, D. R. Chowdhury, S. Ramani, M. T. Reiten, S. N. Luo, A. J. Taylor, H. T. Chen, Experimental demonstration of terahertz metamaterial absorbers with a broad and flat high absorption band, Opt Lett. 15;37:154-156(2012).

[8] S. Bhattacharyya, S. Ghosh, K. V. Srivastava, "Triple Band Polarization-Independent Metamaterial Absorber with Bandwidth Enhancement at X-band," Journal of Applied Physics, 114, 094514, 2013. [9] S. Bhattacharyya, K. V. Srivastava, "Ultra Thin Metamaterial Absorbers using Electric Field Driven LC (ELC) Resonator Structure," in Progress In Electromagnetics Research Symposium, 314-317, 2012

[10] S. Ghosh, S. Bhattacharyya, K. V. Srivastava, "Design of a Bandwidth-Enhanced Ultra Thin Metamaterial Absorber," in Progress In Electromagnetics Research Symposium, 1097-1101, 2013

[11] Y. Avitzour, Y. A. Urzhumov, G. Shvets, "Wide-angle infrared absorber based on a negative-index plasmonic metamaterial", Phys. Rev. B 2009, $79,45131$.

[12] Y. Wang, T. Sun, T. Paudel, Y. Zhang, Z. Ren, K. Kempa, "Metamaterial-plasmonic absorber structure for high efficiency amorphous silicon solar cells" Nano Lett. 12, 440 (2011). 
[13]. J. Hao, L. Zhou, and M. Qiu, "Nearly total absorption of light and heat generation by plasmonic metamaterials” Phys. Rev. B, 83, 165107 (2011).

[14] J. Wang, Y. Chen, J. Hao, M. Yan, and M. Qiu, "Shape-dependent absorption characteristics of three-layered metamaterial absorbers at near-infrared”. Jpn. J. Appl. Phys. 109, 074510 (2011).

[15] X. Liu, T. Tyler, T. Starr, A. F. Starr, N. M. Jokerst, W. J. Padilla, "Taming the blackbody with infrared metamaterials as selective thermal emitters "Phys. Rev. Lett. 107, 045901 (2011).

[16] X. Liu, T. Starr, A. F. Starr, and W. J. Padilla, "Taming the blackbody with infrared metamaterials as selective thermal emitters ", Phys. Rev. Lett. 104, 207403 (2010).

[17]S. Bhattacharyya, K. Va. Srivastava, "Dual Layer Polarization Insensitive Dual Band Metamaterial Absorber with Enhanced Bandwidths," in IEEE Asia Pacific Microwave Conference (APMC) 2014, pp. 816-818, Sendai, Japan, 4-7 November, 2014.

[18] K. Aydin , V. E. Ferry, R. M. Briggs, H. A. Atwater,"Broadband polarization-independent resonant light absorption using ultrathin plasmonic super absorbers", NATURE COMMUNICATIONS, 2:517(2011)

[19] J. M. Hao, L. Zhou, M. Qiu,"Nearly total absorption of light and heat generation by plasmonic metamaterials", PHYSICAL REVIEW B 83, 165107 (2011) 
[20] Y. Wang, T. Y. Sun, T. Paudel, Y. Zhang, Z. F. Ren, K. Kempa, "Metamaterial-Plasmonic Absorber Structure for High Efficiency Amorphous Silicon Solar Cells", Nano Lett. 2012, 12, 440-445

[21] L. Huang, D. R. Chowdhury, S. Ramani, M. T. Reiten, S. N. Luo, A.

J. Taylor, H. T. Chen, "Experimental demonstration of terahertz metamaterial absorbers with a broad and flat high absorption band", Optics Letters 37(2):154-6

[22] S. L. H. F. Ding, L. Mo, F. L. Bao,"Light Absorber with an Ultra-Broad Flat Band Based on Multi-Sized Slow-Wave Hyperbolic Metamaterial Thin-Films", Progress In Electromagnetics Research, 147, $69-79,2014$

[23] B. Zhang, Y. Zhao, Q. Hao, B. Kiraly, I. Khoo, S. Chen, T. J. Huang, "Polarization-independent dual-band infrared perfect absorber based on a metal-dielectric-metal elliptical nanodisk array" Opt. Express 19, 15221 (2011).

[24]. S. Chen, H. Cheng, H. Yang, J. Li, X. Duan, C. Gu, J.Tian, "Polarization insensitive and omnidirectional broadband near perfect planar metamaterial absorber in the near infrared regime" Appl. Phys. Lett. 99, 253104 (2011).

[25] S. Chen, H. Cheng, H. Yang, J. Li, X. Duan, C. Gu, J. Tian, "Polarization insensitive and omnidirectional broadband near perfect 
planar metamaterial absorber in the near infrared regime", Appl. Phys. Lett. 2011, 99, 253104-253108.

[26] H. M. Lee, J. C. Wu, "A wide-angle dual-band infrared perfect absorber based on metal-dielectric-metal split square-ring and square array”, J. Phys. D: Appl. Phys. 45:205101- 205104 (2012)

[27] K. Chen, R. Adato, H. Altug, “ Dual-band perfect absorber for multispectral plasmon-enhanced infrared spectroscopy", ACS Nano. 6,7998-8006 (2012).

[28] N. Zhang, P. Zhou, D. Cheng, X. Weng, J. Xie, L. Deng. "Dual-band absorption of mid-infrared metamaterial absorber based on distinct dielectric spacing layers", Opt. Lett. 38,1125-1127 (2013). [29] M. Zhong, Y. H. Ye. "Role of coupling of localized surface plasmon modes in transmission properties of compound structure metamaterials", Optics Communications, 335, 94-98 (2015).

[30] M. A. Ordal, L. L. Long, R. J. Bell, S. E. Bell, R. R. Bell, R. W. Alexander, C. A. Ward, Appl. Opt. 1983, 22, 1099-1119.

[31] Y . Avitzour , Y . A. Urzhumov , G. Shvets, "Wide-angle infrared absorber based on a negative-index plasmonic metamaterial “. Phys. Rev. B, 79, 045131-045135 (2009)

[32] E. D. Palik. Handbook of Optical Constants in Solids (Academic, 1991). 
[33] Y. L. Hua. Z. Y. Li. "Analytic modal solution to transmission and collimation of light by one-dimensional nano-structured subwavelength metallic slits", J. Appl. Phys. 105, 013104-013107 (2009).

[34] D. R. Smith, S. Schult, P. Markos, C. M. Soukoulis, "Determination of effective permittivity and permeability of metamaterials from reflection and transmission coefficients". Phys. Rev. B 65 (2002) [35] P. Ding, E. J. Liang, G. W. Cai, W. Q. Hu, C. Z. Fan, and Q. Z. Xue, "Dual-band perfect absorption and field enhancement by interaction between localized and propagating surface plasmons in optical metamaterials," J. Opt. 13, 075005 (2011)

[36] D. R. Smith, D. C. Vier, T. Koschny, C. M. Soukoulis, "Electromagnetic parameter retrieval from inhomogeneous metamaterials ”, Phys. Rev. E. 71 036617(2005) [37] X. L. Liu, T. Starr, A. F. Starr, W. J. Padilla, Infrared Spatial and Frequency Selective Metamaterial with Near-Unity Absorbance, Physical Review Letters, 104: 207403-207406(2010)

[38] V. E. Ferry, J. N. Munday, and H. A. Atwater, "Design considerations for plasmonic photovoltaics," Adv. Mater.(Deerfield Beach Fla.) 22(43), 4794-4808 (2010).

[39]X. L. Liu, T. Tyler, T. Starr, A. F. Starr, N. M. Jokerst, and W. J. Padilla, "Taming the blackbody with infrared metamaterials as selective thermal emitters," Phys. Rev. Lett. 107(4), 045901 (2011). 
[40] H. Tao, C. M. Bingham, A. C. Strikwerda, D. Pilon, D.

Shrekenhamer, N. I. Landy, K. Fan, X. Zhang, W. J. Padilla, R. D.

Averitt, Phys. Rev. B, 2008, 78, 241103 